\title{
Desflurane does not decrease myocardial injury in trans-catheter aortic valve implantation with trans-femoral approach under general anaesthesia.
}

\author{
Kenta Okitsu, Takeshi Iritakenishi, Yuji Fujino
}

Department of Anesthesiology and Intensive Care Medicine, Osaka University

\begin{tabular}{|lcccc|} 
& & Group D & Group P & \\
& $\mathbf{N}=\mathbf{2 6}$ & $\mathbf{N}=64$ & $\mathbf{P}$ \\
\hline Peak CK-MB & $\mathrm{9} .25$ & 8.7 & \\
& $\mathrm{ng} / \mathrm{mL}$ & {$[1.3-20.5]$} & {$[2.6-49.7]$} & 0.52 \\
\hline Peak Troponin I & $\mathrm{ng} / \mathrm{ml}$ & $\begin{array}{c}1.23 \\
{[0.088-6.4]}\end{array}$ & $\begin{array}{c}1.24 \\
{[0.036-29]}\end{array}$ & 0.71 \\
\hline $\begin{array}{l}\text { Postoperative } \\
\text { Myocardial injury * N (\%) }\end{array}$ & $20(76.9)$ & $44(68.8)$ & 0.61 \\
\hline
\end{tabular}

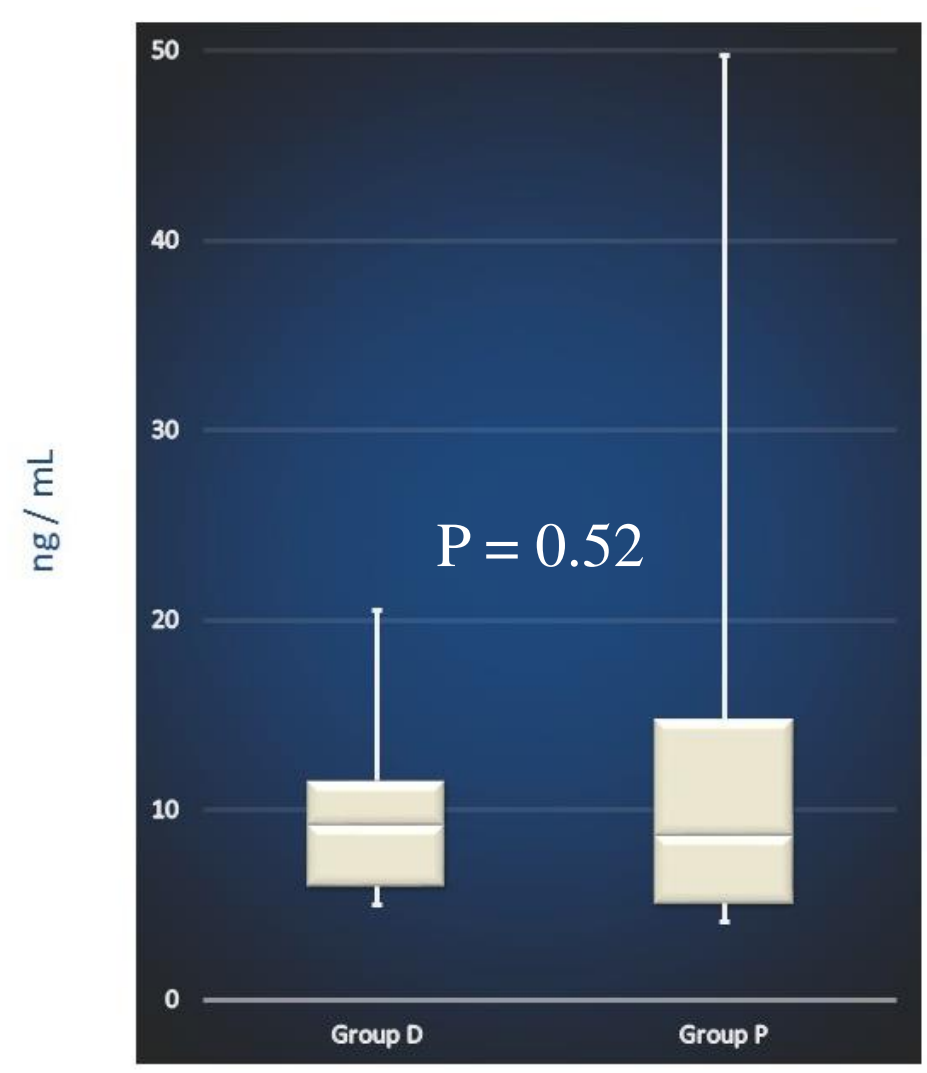

Peak CK-MB

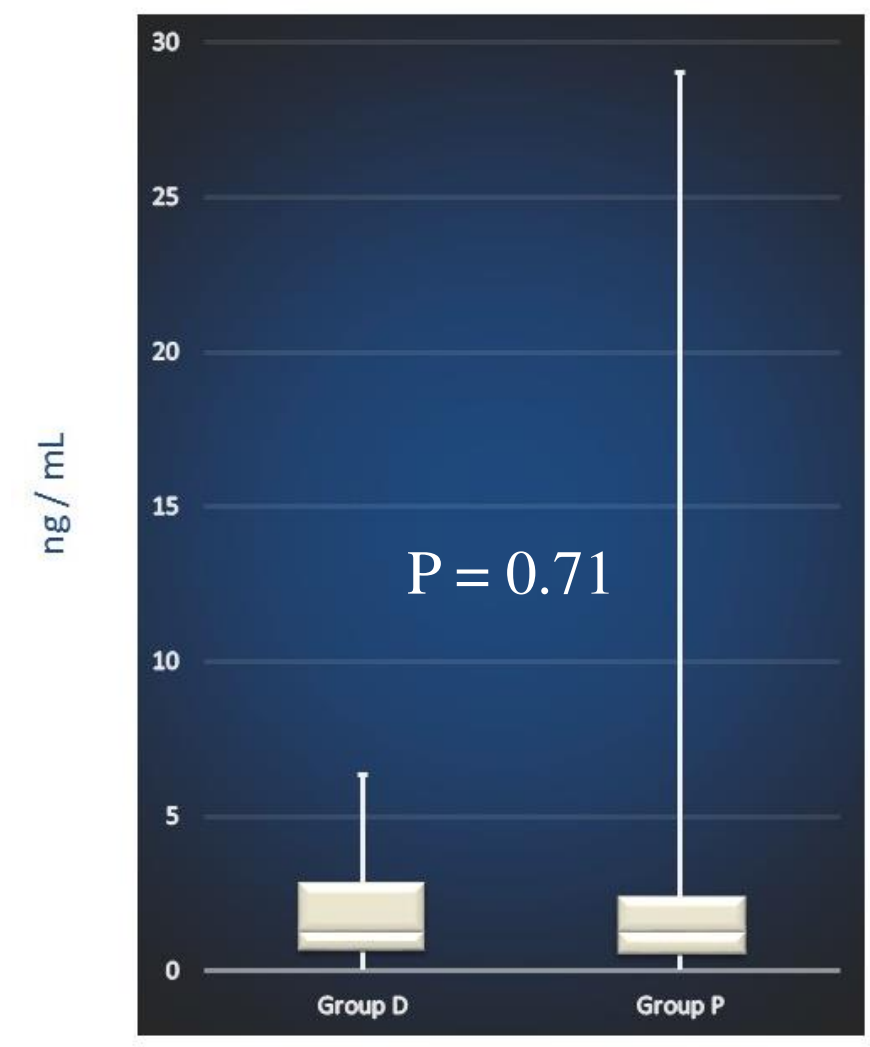

Peak Troponin I either CK-MB $>5$ times or Troponin I $>15$ times the upper reference limits within postoperative $72 \mathrm{~h}$

\section{Background}

Cardio-protective effect of volatile anaesthetics is shown in cardiac surgery, for example, on-pump CABG.

(Ref. "Cardiac troponins and volatile anaesthetics in coronary artery bypass graft surgery." Eur J Anaesthesiol 2016; 33:396-407)

However, it is unclear whether volatile anaesthetics decrease the risk of postoperative myocardial injury in patients undergoing trans-catheter aortic valve implantation (TAVI).

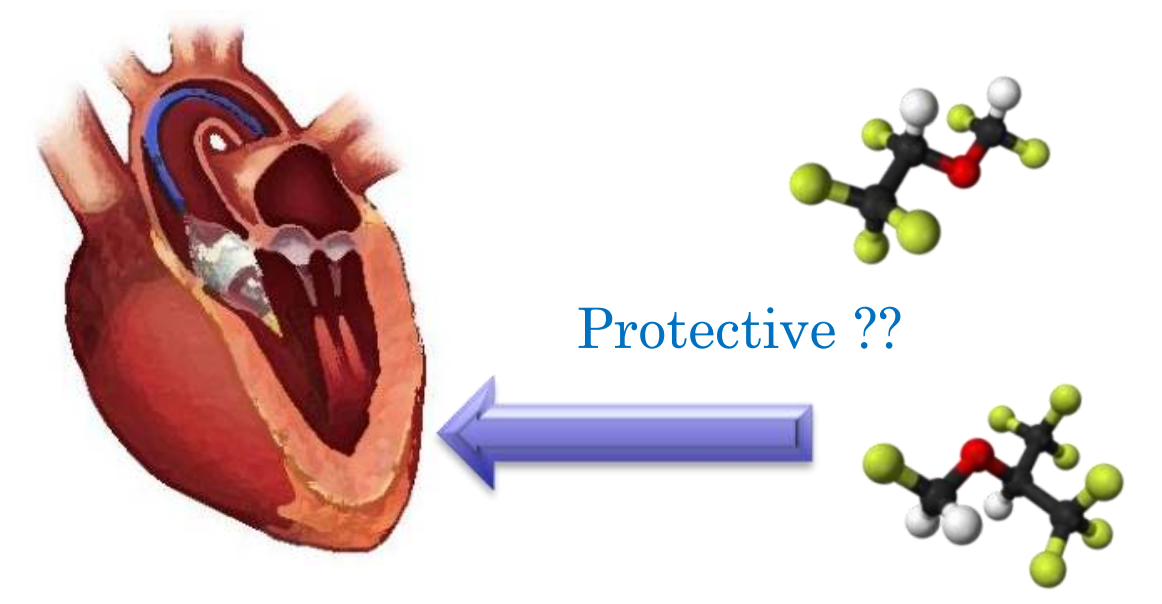

Materials and Methods

Settings: Retrospective study in a single university hospital

Inclusion: Adult patients undergoing TAVI with femoral approach between January 2015 and September 2016 (100 cases)

Exclusion: Patients who underwent TAVI and other procedure at the same time (5 cases)

Patients who required cardio-pulmonary bypass during the procedure (5 cases)

Allocation: Group D; Patients who were maintained general anaesthesia with desflurane $(\mathrm{n}=26)$

Group P; Patients who were maintained general anaesthesia with propofol $(\mathrm{n}=64)$

Primary Outcome: The rate of patients who developed postoperative myocardial injury defined as increase in myocardial biomarker including CK-MB and Troponin I

Secondary Outcomes: Peak CK-MB and troponin I value within $72 \mathrm{~h}$ Statistical Analysis: The rate of postoperative myocardial injury was compared using Fisher's exact test and Peak CK-MB and troponin I value were compared using Wilcoxon rank sum test between groups

\begin{tabular}{|l|c|c|c|}
\hline & Group D $(\mathrm{n}=26)$ & Group P $(\mathrm{n}=64)$ & $\rho$ \\
\hline Age & $82.3 \pm 6.2$ & $83.6 \pm 6.2$ & 0.29 \\
\hline Sex Male / Female & $7 / 19$ & $15 / 49$ & 0.79 \\
\hline Height cm & $150.9 \pm 8.8$ & $150.2 \pm 8.2$ & 0.73 \\
\hline Weight kg & $51.4 \pm 9.2$ & $50.0 \pm 11.2$ & 0.29 \\
\hline LVEF \% & $65.2 \pm 13.4$ & $63.5 \pm 12.6$ & 0.44 \\
\hline Aortic Valve Area cm2 & $0.67 \pm 0.14$ & $0.71 \pm 0.18$ & 0.35 \\
\hline Mean Pressure Gradient mmHg & $51.2 \pm 21.5$ & $47.3 \pm 16.2$ & 0.43 \\
\hline Coronary Artery Disease $\mathrm{n}(\%)$ & $8(30.8)$ & $25(39.1)$ & 0.63 \\
\hline COPD n (\%) & $2(7.7)$ & $5(7.8)$ & 1.0 \\
\hline Diabetes mellitus n (\%) & $6(23.1)$ & $21(32.8)$ & 0.45 \\
\hline Preoperative Hemoglobin g/dl & 11.51 .3 & 11.41 .6 & 0.72 \\
\hline Preoperative Creatinine mg/dl & $0.97[0.47-12.7]$ & $0.89[0.52-10.7]$ & 0.84 \\
\hline $\begin{array}{l}\text { Devices } \\
\text { SAPIEN XT or SAPIEN3 / } \\
\text { Core Valve / LOTUS }\end{array}$ & $37 / 21 / 6$ & $20 / 5 / 1$ & 0.23 \\
\hline
\end{tabular}

LVEF: left ventricular ejection fraction COPD: chronic obstructive pulmonary disease

Results

We could not find any difference regarding patients background (above Table) as well as the occurrence of postoperative myocardial injury and peak myocardial biomarkers (headline Table and Figures) between groups.

\section{Limitations}

First, small and asymmetric study population in Group D. Second, we could not obtain postoperative electric cardiographies and there was no symptomatic myocardial infarction in patients included in our study, therefore we evaluated postoperative myocardial injury only using myocardial enzymes.

\section{Conclusions}

We demonstrated that desflurane does not decrease myocardial injury compared with propofol in trans-femoral TAVI under general anaesthesia. Future randomized controlled trials are required to confirm our findings. 